\title{
Binocular rivalry with chromatic contours
}

\author{
PEEP F. M. STALMEIER and CHARLES M. M. DE WEERT \\ University of Nijmegen, Nijmegen, The Netherlands
}

\begin{abstract}
The contribution of colored contours to binocular rivalry was investigated. Orthogonal circular and radial figures were used as stimuli. Binocular rivalry was measured between a fixed achromatic target presented to the left eye and a two-color target presented to the right eye. Various color combinations were presented to the right eye to assess the rivalry strength of these color combinations. The main conclusion is that the contributions of color contours in binocular rivalry and minimally distinct border experiments are closely related; that is, signals from shortwavelength-sensitive cones do not make an appreciable contribution to rivalry.
\end{abstract}

Levelt (1968) concluded that binocular rivalry is due mainly to the presence and amount of nonfusionable contours in each eye (contour effect). In an informal experiment, he showed that purely chromatic (isoluminant) red-white contours led to similar effects as luminance contours did. To obtain more detailed knowledge about the contribution of colored contours to binocular rivalry, we studied the course of the binocular rivalry process when the color contours were changed in a quantitative manner. The data obtained with this method might be relevant for the issue of large color differences. If the rivalry process varies with the color distance between the two colors forming the contour, then rivalry might be used to quantify combined chromatic and luminance differences. The advantage of this method would be that quite large color differences could be measured without asking the subject for direct color difference judgments.

Ward and Boynton's (1974) experiments are of particular interest, given the importance of the contour effect in binocular rivalry. In their minimal distinct border (MDB) experiments, subjects rated the strength of a chromatic border between two juxtaposed color fields with reference to a variable achromatic contour. Tansley and Boynton (1976) found indications in multidimensional scaling analysis of MDB experiments that colors along tritanopic confusion lines essentially collapse on the spectral locus. This means that in the MDB task, differential stimulation of the blue cone is far less effective than differential stimulation of the red and green cones (Kaiser \& Boynton, 1985; Valberg \& Tansley, 1977). We suspected that these properties of color contours in MDB experiments are also important for binocular rivalry.

The Dutch Organization for the Advancement of Pure Science is gratefully acknowledged for funding this project. This research was conducted while P. F. M. Stalmeier was supported by PSYCHON Grant 560-262021 of this organization, awarded to C. M. M. de Weert. We thank Mary Hayhoe and Patrick Bossuyt for helpful discussions. Address correspondence to Peep F. M. Stalmeier, University of Nijmegen, Department of Experimental Psychology, P.O. Box 9104, 6500 HE Nijmegen, The Netherlands.
Experiments 1 and 2 of the present study were concerned with the effect of color contours in binocular rivalry. An attempt was made in Experiment 3 to express the color contour strength in binocular rivalry in terms of an equivalent achromatic contrast strength.

\section{GENERAL METHOD}

\section{Subjects}

Six subjects, 5 males and 1 female, participated in these experiments. They ranged in age from 26 to 43 years. Three of them were corrected myopes. The subjects were color normal, as determined with the Ishihara color test and the Farnsworth-Munsell 100-hue test. Four paid subjects were naive and had no previous experience with binocular rivalry measurements. The 6 subjects were selected out of 9 subjects on the following criteria: maximum reproducibility of dominance values on a red-green test stimulus and, at worst, composites were perceived less than $40 \%$ of the measurement time.

\section{Apparatus \\ Stimuli were presented on a high-resolution color monitor (Barco CTVM 2/51 H) with 10-bit-wide red, green, and blue inputs. The monitor was coupled with a DEC PDP 11/23 computer (Wittebrood, Wansink, \& de Weert, 1981). We used computer programs that enable the luminance of a color to be changed while the chromatic- ity coordinates of the color remain fixed, and programs that allow for variation along a straight line in the combined luminance and chromaticity space. \\ Stimulus display and data acquisition were under computer control.}

\section{Stimulus Configuration}

Figure 1 shows the stimulus pair presented to the left and right eyes. One stimulus consisted of concentric circles; the other consisted of radials. Table 1 contains the luminance levels, as measured with a Pritchard SPECTRA photometer. These particular stimuli were chosen instead of the more common orthogonal oblique gratings or horizontal/vertical pair of gratings because the more common stimuli can give rise to more eye-following motions that in particular weaken one stimulus and strengthen the other.

In the circular figure, presented to the left eye, achromatic colors were used. The Michelson contrast, defined as $K=$ $2 \times\left(\mathrm{L}_{\max }-\mathrm{L}_{\min }\right) /\left(\mathrm{L}_{\max }+\mathrm{L}_{\min }\right)$, was equal to 0.5 . $\mathrm{L}_{\max }$ and $\mathrm{L}_{\min }$ are the local luminance levels as given in Table 1 . The space-averaged luminance of the circular figure was equal to $12 \mathrm{~cd} / \mathrm{m}^{2}$. In the segmented figure, presented to the right eye, different pairs of colors 


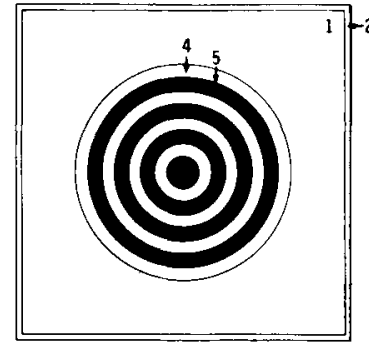

LEFT EYE

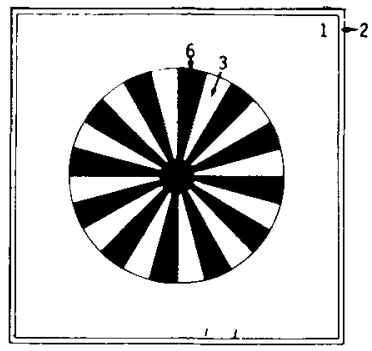

RIGHT EYE
Figure 1. Stinulus pair used for binocular rivalry. The concentric circular figure was always presented to the left eye, the segmented radial figure to the right eye.

were presented in the segments. A central spot of the same size and color used in the circular figure was added to the segmented figure. It provided a steady fixation point, enhancing the fusional stability of the stimuli. Moreover, it set a limit to the high-frequency content of the segmented figure.

The contour lengths in the left and right figures were approximately equal. Both the circular and segmented figures subtended visual angles of $1.48^{\circ}$. The viewing distance was $3.1 \mathrm{~m}$. A mirror stereoscope was used to combine the left and the right figures dichoptically. A septum separated the two figures.

\section{Procedure}

In the segmented figure, pairs of colors were presented in the radial segments. If we could assume that both eyes have equal spectral sensitivity curves, it would not matter to which eye the colors are presented; however, because sensitivity differences frequently do occur, we chose to present the colored figure always to the right eye. The colors used in Experiments 1 and 2 were made isoluminant to white (chromaticity coordinates: $0.33,0.33$ ) with a flicker photometric calibration procedure (the flicker rate was $12.5 \mathrm{~Hz}$ ) with a $1^{\circ}$ circular field, in which the subjects used the right eye only. This was done for each subject. Thus every subject had his/her own set of isoluminant colors. The circular figure in the left eye remained unchanged during all three experiments. Differences in dominance can therefore be attributed only to the variation of colors in the segmented figure.

Time registration of the binocular rivalry process during 1-min trials was done with two switches. The subjects were instructed to fixate the central spot and to depress the left or right switch, respectively, whenever the circular or segmented figure was completely visible. Composites were perceived but not registered as a separate category, since we were interested only in the complete dominance of one figure or the other. The total time of occurrence of composites may be derived from the data if necessary.

After each $1 \mathrm{~min}$ of measured rivalry for a given color combination, there was a brief rest for $30 \mathrm{sec}$. Usually, about 10 consecutive trials were given, followed by a rest period of at least $3 \mathrm{~min}$. This schedule was not too tight, and the subjects were urged to pause whenever they wanted. Each session was finished after $1 \mathrm{~h}$, dur-

Table 1

CIE (xy) Coordinates of the Stimulus Colors

\begin{tabular}{cccc}
\hline Color No. & $x$ & $y$ & \multicolumn{1}{c}{ L } \\
\hline 1 & .33 & .33 & 0.29 \\
2 & .33 & .33 & 0.49 \\
4 & .33 & .33 & 14.60 \\
5 & .33 & .33 & 8.75 \\
3,6 & variable & & \\
\hline
\end{tabular}

Note-The stimulus background was dark. $\mathrm{L}=$ luminance (in $\mathrm{cd} / \mathrm{m}^{2}$ ). ing which time an average of 35 combinations were measured. Occasionally, two sessions were held on one day. Every color combination was repeated 10 times. All combinations were presented in a different random order for each subject. A chinrest was used for the fixation of the subjects' heads.

Before each session, the convergence of the color monitor was carefully controlled to ensure that no dividing line was visible between the colored segments.

From a 1-min trial, seven parameters were computed and stored on file: $n_{1}$ and $n_{\mathrm{r}}$, the number of times the left or the right stimulus dominated; $t_{1}$ and $t_{r}$, the average duration of the left or the right stimulus; $s_{1}$ and $s_{\mathrm{r}}$, the standard deviations of these durations; and $D$, the dominance measure, defined as $\left(T_{\mathrm{r}}-T_{1}\right) /\left(T_{\mathrm{r}}+T_{\mathrm{i}}\right) . T_{\mathrm{r}}$ and $T_{1}$ are the total times that the right and left stimulus were visible, that is, $T_{\mathrm{r}}=n_{\mathrm{r}} \times t_{\mathrm{r}}$ and $T_{1}=n_{1} \times t_{1}$.

\section{EXPERIMENT 1 DOMINANCE OF COLOR PAIRS OF SEVEN COLORS}

In this experiment, dominance was measured for each combination of seven colors, distributed over that area of the CIE chromaticity space that is covered by our color monitor. The colors were made isoluminant at $12 \mathrm{~cd} / \mathrm{m}^{2}$ for each subject, using the flicker procedure described above. Thus we hoped to avoid the contribution of a luminance component to the contour and to obtain purely chromatic contours. Table 2 and Figure 2 show the location on the CIE $(x, y)$ diagram of the colors used in this experiment.

In the first series, all 15 possible color combinations of the first six colors were presented in the segmented figure to the 6 subjects. The mean data of 5 subjects are presented in Figure 3 (open circles). The dominance of a color pair is plotted as a function of the distance between these colors in the CIE $1960(u, v)$ diagram. The CIE $(u, v)$ color-order system is intended to be perceptually uniform for small color differences (Wyszecki \& Stiles, 1983). Positive dominance values indicate predominance of the segmented figure over the circular figure. (For clarity, the data points are connected by lines.) The numbers near the data points denote the colors presented in the segmented figure in accordance with the color numbers given in Table 2. The standard deviation of $D$ for the 10 repeated measurements of a given color pair was typically between 0.07 and 0.2 units of $D$ for individual subjects.

To assess the intraobserver reproducibility and the reliability of the measurements, this experiment was repeated with all seven colors (see T'able 2). Thus dominance values of 21 color combination: were measured in the second series. The time interval beiween these two series was approximately 2.5 months. Four subjects, the same as in the first series, participated. The mean data of 3 subjects are shown in Figure 3 (closed circles). They are shifted upward 0.2 units of $D$ for clarity.

For every subject, the color combinations were ordered with respect to increasing dominance value, and Spearman's coefficient of rank correlation was calculated between these two orderings of the first and second series. 
Table 2

CIE $(x, y)$ Coordinates of the Colors Used in Experiment 1

\begin{tabular}{cccc}
\hline Color No. & $x$ & $y$ & L \\
\hline 1 & 0.30 & 0.55 & 12 \\
2 & 0.41 & 0.47 & 12 \\
3 & 0.60 & 0.33 & 12 \\
4 & 0.20 & 0.10 & 12 \\
5 & 0.35 & 0.19 & 12 \\
6 & 0.25 & 0.33 & 12 \\
7 & 0.33 & 0.33 & 12 \\
\hline
\end{tabular}

Note-Color numbers are the same as those in Figure 2. $\mathrm{L}=$ luminance (in $\mathrm{cd} / \mathrm{m}^{2}$ ).

For the subjects J.S., G.W., E.H., and P.S., these coefficients are $0.75,0.83,0.94$, and 0.86 , respectively. In Table 3 rank correlation coefficients between subjects are given. Note that the data for Subject E.H. do not agree with those of the other subjects; therefore, we have excluded his results from the averaged data, presented in Figure 3, although he was not excluded from subsequent analyses.

Figure 3 clearly shows the existence of a saturation plateau for large values of $d(u, v)$. $D$ seems to increase with increasing CIE $(u, v)$ distance, but not monotonically. Closer inspection of Figure 3 shows that the dips in the curves occur for color pairs consisting of colors along lines pointing more or less to the tritanopic confusion point. Only the data of Subject E.H. show an exception to this general decrease of dominance values for color pairs along tritanopic confusion lines.

The saturation plateau for most subjects in the second series is generally not at the same level as that in the first series; that is, there is a drift of the dominance values over time. This means that dominance values can be compared only within one series.

Some of the isoluminant color combinations-for example, $(1,6)$ and $(2,7)$-showed extremely weak chromatic contours. This phenomenon has been described previously (Gregory, 1977; Koffka \& Harrower, 1931). It supports our claim that these colors were isoluminant. Tansley and Boynton (1978) proposed that such blurred contours occur when colors are closely set along tritanopic confusion lines. This is in accordance with our results.

We roughly compared our data for the series with seven colors to the MDB equivalent achromatic contrast settings given by Ward and Boynton (1974), who used spectral colors only. We selected their settings for the spectral colors closest to the dominant wavelengths of our colors. Color 5 was excluded since Ward and Boynton did not measure any purple. Note that the correspondence between the colors is not very good; for example, the color combinations $(1,6),(4,7)$, and $(2,4)$ are almost tritanopic in our color set, whereas Ward and Boynton's spectral counterparts are not. Pearson's product-moment correlation coefficients were calculated in two ways. First, we correlated individual color combinations; that is, we correlated the dominance values of each color combination (see Figure 3, closed points, color 5 excluded) with the achromatic contrast equivalents of the corresponding
MDB color pairs. The correlation coefficient $r(13)=0.5$, which is significant at only the $10 \%$ level (two-tailed test). Second, we correlated the overall strength of a color. For every color, we added the dominance values of its combinations with the remaining five colors to evaluate the overall strength of that color. We did the same for the MDB contrast equivalents. This had the desired effect of averaging across the orientations of the color pairs in color space. The correlation coefficient $r(4)=0.82$, which is just significant at the $5 \%$ level (two-tailed test). This corre-

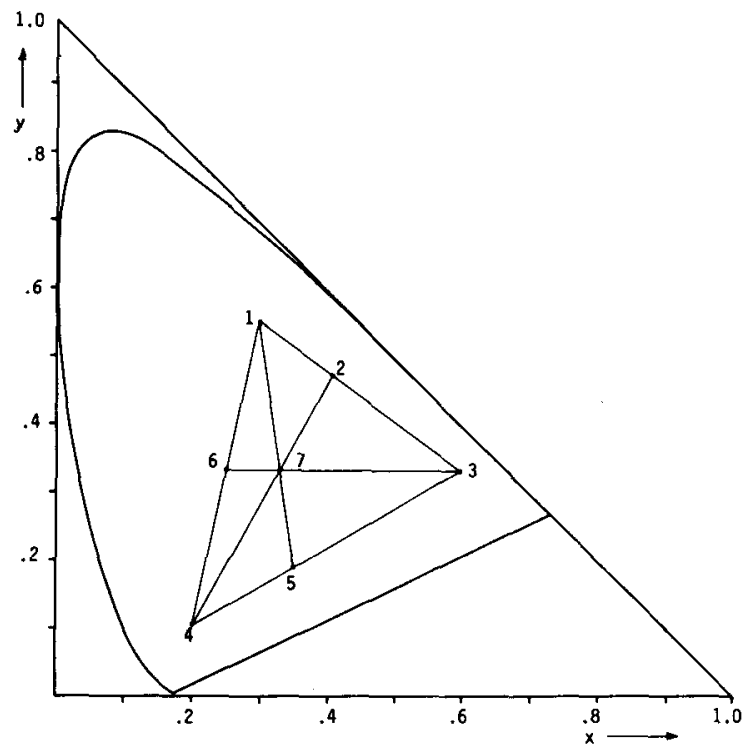

Figure 2. Location in the CIE $(x, y)$ diagram of the colors used in Experiment 1.

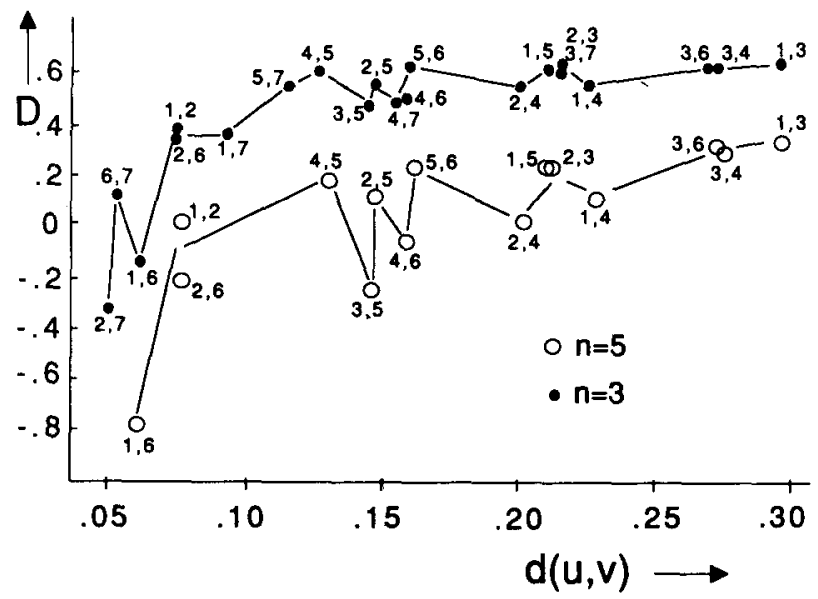

Figure 3. Mean dominance values of color pairs in the segmented figure plotted against the distance of the colors in the CIE $1960(u, v)$ diagram. Open circles correspond to the mean data of 5 subjects for the color set containing six colors. Closed circles correspond to the mean data of 3 subjects for the color set containing seven colors. The closed circles are shifted upward 0.2 units of $D$ for clarity. Positive values indicate predominance of the segmented figure over the circular figure. Numbers near the data points denote the colors as given in Table 2. 
Table 3

Spearman's Coefficients of Rank Correlation Among

Subjects for the Series with 6 (15 Combinations;

Top) and 7 (21 Combinations; Bottom) Colors

\begin{tabular}{lcccccc}
\hline & P.S. & J.S. & G.W. & E.H. & R.W. & C.W. \\
\hline P.S. & - & 0.66 & 0.73 & 0.33 & 0.71 & 0.82 \\
J.S. & & - & 0.60 & 0.60 & 0.70 & 0.53 \\
G.W. & & & - & 0.15 & 0.80 & 0.89 \\
E.H. & & & & - & 0.25 & -0.01 \\
R.W. & & & & & - & 0.83 \\
C.W. & & & & & & - \\
P.S. & - & 0.77 & 0.87 & 0.50 & & \\
J.S. & & - & 0.83 & 0.57 & & \\
G.W. & & & - & 0.45 & & \\
E.H. & & & & - & & \\
\hline
\end{tabular}

Table 4

CIE $(x, y)$ Coordinates of the Colors Used in Experiment 2

\begin{tabular}{cccc}
\hline Color No. & $x$ & $y$ & L \\
\hline 1 & 0.550 & 0.359 & 12 \\
2 & 0.450 & 0.264 & 12 \\
3 & 0.400 & 0.217 & 12 \\
4 & 0.375 & 0.193 & 12 \\
5 & 0.395 & 0.465 & 12 \\
6 & 0.330 & 0.330 & 12 \\
7 & 0.300 & 0.268 & 12 \\
8 & 0.235 & 0.133 & 12 \\
9 & 0.450 & 0.275 & 12 \\
10 & 0.400 & 0.300 & 12 \\
11 & 0.330 & 0.335 & 12 \\
12 & 0.260 & 0.370 & 12 \\
\hline
\end{tabular}

Note-Color numbers are the same as those in Figure 4. $\mathrm{L}=$ luminance (in $\mathrm{cd} / \mathrm{m}^{2}$ ).

lation is quite good in view of the fact that we used nonspectral colors, and it demonstrates that the contour strength of colored contours in binocular rivalry and MDB are closely related to each other.

\section{EXPERIMENT 2 DOMINANCE OF COLORS ALONG CRITICAL AXES}

In Experiment 1 we found indications that the dominance of a stimulus consisting of colors positioned along tritanopic confusion lines is relatively low. We dealt with this finding in more detail in Experiment 2. Isoluminant colors were chosen on two tritanopic confusion lines and on a line along which the B-cone excitation level is constant (Boynton \& Kambe, 1980). Again, these colors were made isoluminant to $12 \mathrm{~cd} / \mathrm{m}^{2}$, using the flicker procedure. We assumed that this would eliminate any luminance difference at the contour. Figure 4 and Table 4 show the colors used in this experiment.

Lines $a$ and $b$ in Figure 4 are tritanopic confusion lines. Along these lines, only the B-cone excitation level changes. Along line c, the B-cone excitation level is constant. On every line, four colors were chosen. This yielded a total of six possible color pairs per line, which were presented in random sequence in the segmented figure in separate series. The dominance of these color pairs was measured by the subjects.

Dominance values, as a function of the distance between two colors in the CIE $1960(u, v)$ diagram, are plotted in Figures $5 \mathrm{a}, 5 \mathrm{~b}$, and $5 \mathrm{c}$. The numbers near the data points denote which color numbers (see Table 4) were presented in the segmented figure. Figure $5 \mathrm{c}$ shows that the variation of the dominance values between the color combinations along line $c$ is not very strong. However, the dominance values for the color combinations along the tritanopic confusion lines (Figures $5 \mathrm{a}$ and $5 \mathrm{~b}$ ) clearly increase with the color distance of the color pair along the tritanopic line.

It is commonly held that the presence of nonfusionable contours gives rise to binocular rivalry. Tansley and Boynton (1976) found in MDB experiments that B cones scarcely participate in border perception, that is, that tritanopic color pairs do not give rise to contour formation. Although the results of Experiment 1 showed that the predominance of stimuli with colors along a tritanopic confusion line is relatively weak, the results in Figures 5a and $5 \mathrm{~b}$ for the two tritanopic lines clearly show that the dominance values increase with increasing distance along tritanopic lines. Thus, there is an apparent contradiction between our finding and the MDB results.

This contradiction is resolved if we use the MDB criterion instead of the flicker criterion for isoluminance. It appeared that these two criteria lead to different luminance levels. ${ }^{1}$ For instance, if the luminances of color pairs $(1,4)$ and $(5,8)$ are carefully adjusted to yield a MDB, their dominance values drop to the same level as the most leftward points in Figures $5 \mathrm{a}$ and $5 \mathrm{~b}$. This was the case for all 3 subjects. Apparently, the luminance mismatch between the MDB and flicker criterion increases

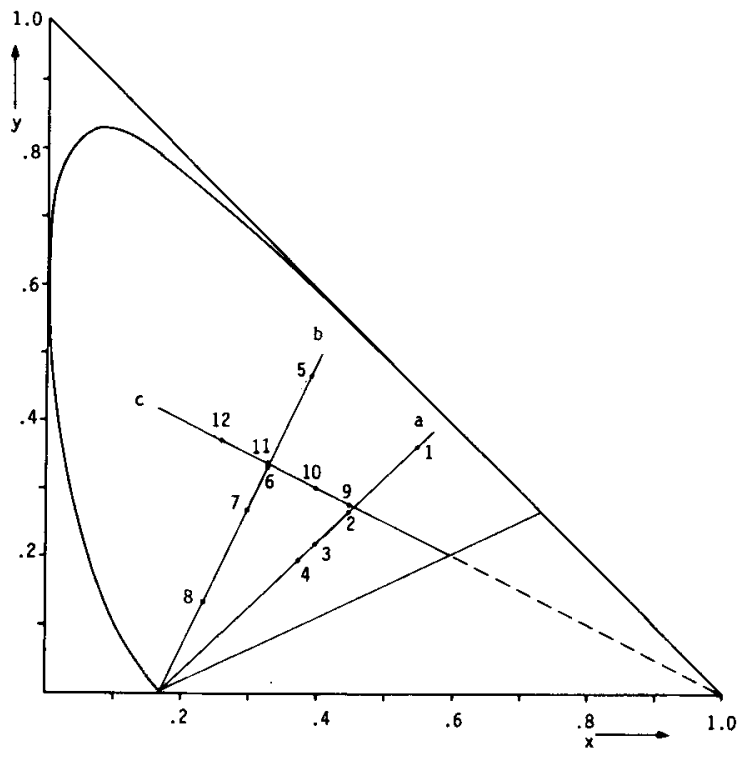

Figure 4. Location in the CIE $(x, y)$ diagram of the colors used in Experiment 2. Lines a and $b$ are tritanopic confusion lines; along line $c$, the B-cone excitation level is assumed to be constant. 

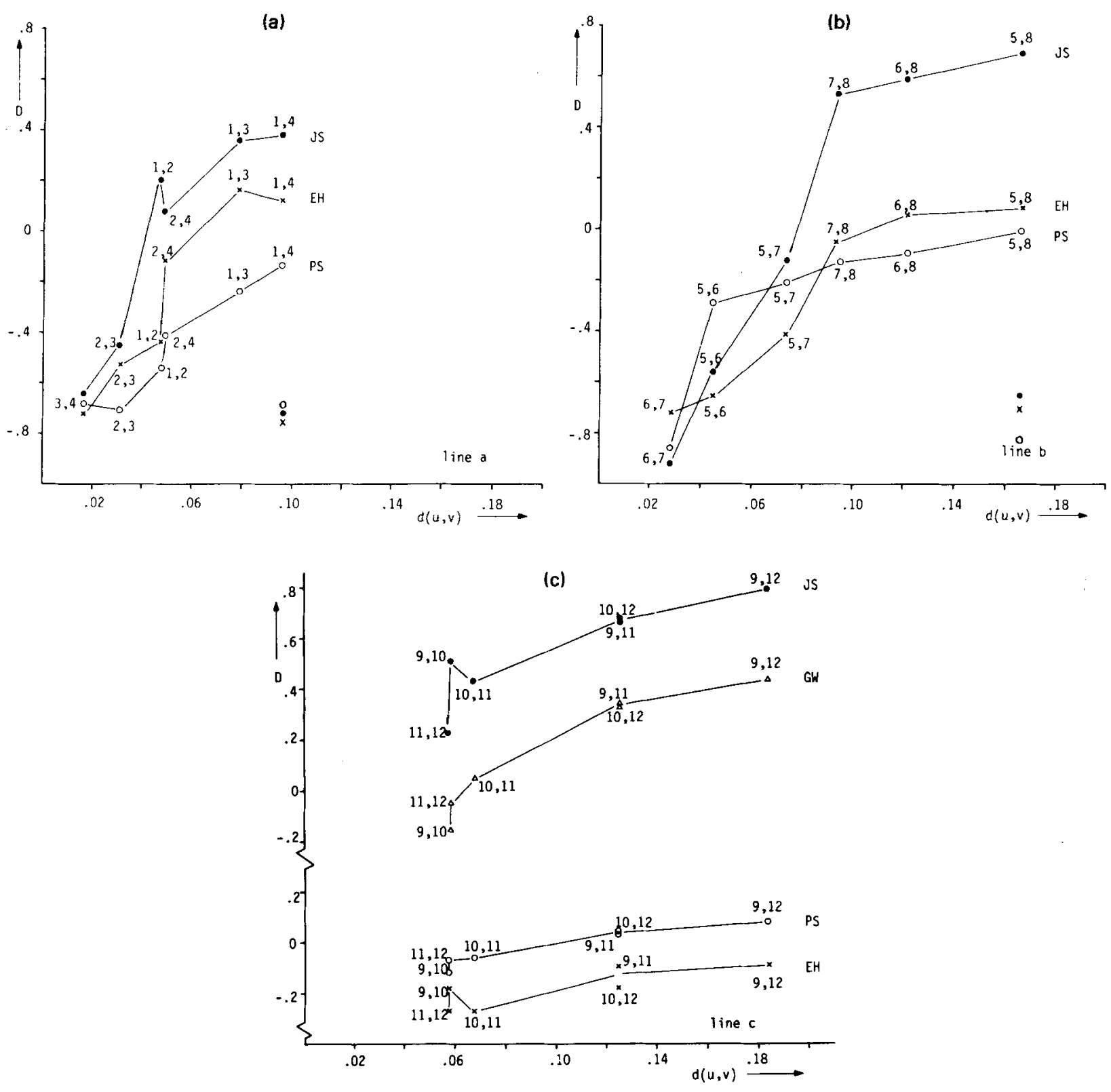

Figure 5. Dominance values of color pairs plotted against the distance of these colors in the CIE $1960(u, v)$ diagram. Colors along lines $a$ and $b$ (Figures 5a and 5b, respectively) are tritanopic confusion pairs. For colors along line $c$ (Figure $5 c$ ), the B-cone excitation level is assumed to be constant. Color pairs connected with lines are made isoluminant with the flicker criterion. The remaining color pairs at the right near the $(u, v)$ axis in Figures $5 \mathrm{a}$ and $5 \mathrm{~b}$ are made isoluminant using the MDB criterion. Figures near the data points denote the colors as given in Table 4.

as the tritanopic difference between the colors increases. We conclude that there is no contour formation for tritanopic color pairs and, consequently, no rivalry, provided that the MDB criterion for isoluminance is used. This finding confirms the close relationship between the contribution of color contours in binocular rivalry and MDB experiments.

\section{EXPERIMENT 3 PURE COLOR CONTRAST VERSUS ACHROMATIC CONTRAST}

The relative strengths of pure (i.e., isoluminant) color and luminance contours have been investigated by several authors. Hilz and Cavonius (1970) found that luminance 
contours are stronger than isoluminant color contours in a grating detection task. They estimated that an achromatic contrast of 0.3 to 0.5 gives rise to the same acuity as the maximum chromatic contrast. Ward and Boynton (1974) found that the maximum equivalent achromatic contrast setting for MDB color borders has an achromatic contrast of 0.4 (recalculated with our contrast definition). In Experiment 3, we measured with binocular rivalry the relative strength of color contours (red-green) and achromatic contours.

The strongest chromatic color combination found in Experiment 1 was red-green. To ensure that this color combination was isoluminant, we presented five red-green combinations with the luminance of red fixed at $12 \mathrm{~cd} / \mathrm{m}^{2}$ and the luminance of green ranging from 9 to $13 \mathrm{~cd} / \mathrm{m}^{2}$, as measured with the Pritchard photometer. Thus we circumvented a MDB isoluminance match for red-green. This match is quite difficult to obtain because the border never disappears. Insofar as red-green MDB matches were made, the green luminance levels were always within the given interval. Furthermore, two achromatic contrasts of 0.4 and 0.87 were presented. The red-green and achromatic combinations were presented at random in one series in the segmented figure. Five subjects participated. The results are shown in Figure 6.

As shown in Figure 6, the differentiation between the various red-green combinations is weak, due to saturation effects. One would expect the presence of a minimum in the dominance values for the red-green combi-

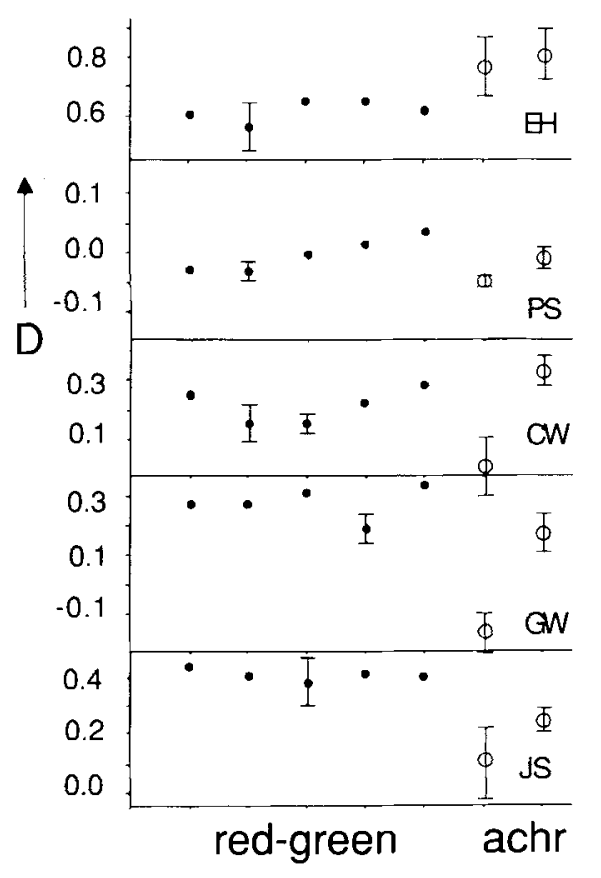

Figure 6. Dominance value of five red-green and two achromatic color pairs used in the segmented figure. From left to right, the closed points correspond to color combinations $\mathbf{R}_{12} \mathbf{G}_{9}, \mathbf{R}_{12} \mathbf{G}_{10}, \mathbf{R}_{12} \mathbf{G}_{11}$, $R_{12} G_{12}$, and $R_{12} G_{13}$. Subscripts denote the luminance level in $\mathrm{cd} / \mathrm{m}^{2}$. Open points correspond to the dominance values for achromatic contrasts of 0.4 and 0.87 . Bars indicate one standard deviation. nations, when the luminances of red and green colors are equal according to the MDB criterion. This minimum is not pronounced, because the red-green border remains quite visible, whatever the relative red-green luminance levels.

The intersubject variability in the results is quite large. Figure 6 shows that the dominance value for the achromatic contrast of 0.87 is well above or at the same level as the minimum of the red-green dominance values, except for Subject J.S. The dominance value of achromatic contrast of 0.4 is below the minimum of the red-green dominance values, except for Subject E.H. We conclude that, given the intersubject variability, no reliable estimate of the achromatic contrast, equivalent to the red-green pure color contrast, can be made. However, our data do not seem to be unreasonable in view of the achromatic contrast values of about 0.4 , suggested by $\mathrm{Hilz}$ and Cavonius (1970) and Ward and Boynton (1974), which were obtained with different experimental methods.

\section{GENERAL DISCUSSION}

In these experiments, we investigated the role of purely chromatic contours in binocular rivalry. The results indicate that colored contours contribute to binocular rivalry in the same way that they contribute to MDB strength; that is, short-wavelength-sensitive cones do not make an appreciable contribution to rivalry. The absence of a MDB for tritanopic color pairs is mirrored by the almost complete absence of rivalry with contours formed by such color pairs, provided a precise isoluminance match using the MDB criterion is made.

Our conclusions agree fully with those arrived at by Rogers and Hollins (1982), who measured binocular rivalry for a series of colored targets in trichromats and dichromats. They found an effect of color on binocular rivalry for trichromats but not for dichromats. From this, they concluded that binocular rivalry is tritanopic. The occasional breakdown of rivalry, anecdotically mentioned for their 453- and 482-nm color pair, also agrees well with the decrease or breakdown of rivalry that we recorded for several tritanopic color pairs. Note that their colors were equated for brightness. The brightness and MDB criterion lead to quite different luminance levels as the saturation of a color increases (Wagner \& Boynton, 1972). This explains that no breakdown of rivalry for other more or less tritanopic color pairs was recorded in their data.

The presented method appears to be inadequate to quantify large color differences beween the two colors forming the chromatic borders in the segmented figure. First, there is the saturation in dominance when the color difference, expressed in CIE $1960(u, v)$ units, becomes larger. This saturation sets in early for colors, discriminated by a Hering type of red-green opponent color channel. Second, the intersubject variability in the relative chromatic contour strengths, measured with binocular rivalry, is quite large, indicating that the method cannot be used for precise quantitative measurements. Finally, neither binocular rivalry nor the MDB method that was proposed 
by Ward and Boynton (1974) for the measurement of large color differences is sensitive to tritanopic color differences.

\section{REFERENCES}

Boynton, R. M., \& Kambe, N. (1980). Chromatic difference steps of moderate size measured along theoretically critical axes. Color Research \& Application, 5, 13-23.

GREGORY, R. L. (1977). Vision with isoluminant colour contrast: 1. A projection technique and observations. Perception, 6, 113-119.

HiLz, R., \& Cavonius, C. R. (1970). Sehschärfe bei Farbunterschieden ohne Helligkeitsunterschiede. Vision Research, 10, 1393-1398.

KAISER, P. K. (1971). Minimally distinct border as a preferred psychophysical criterion in visual heterochromatic photometry. Joumal of the Optical Society of America, 61, 966-971.

KAISER, P. K., BoYNTON, R. M. (1985). Role of the blue mechanism in wavelength discrimination. Vision Research, 25, 523-529.

KoffKa, K., \& Harrower, M. R. (1931). Beiträge zur Psychologie der Gestalt. Psychologische Forschung, 15, 193-275.

Levelt, W. J. M. (1968). On binocular rivalry (Minor Series 2. Psychological studies). The Hague, Netherlands: Mouton.

Rogers, D. C., \& Hollins, M. (1982). Is the binocular rivalry mechanism tritanopic? Vision Research, 22, 515-520.

Tansley, B. W., Boynton, R. M. (1976). A line, not a space, represents visual distinctness of borders formed by different colors. Science, 191, 954-957.

TANSLEY, B. W., \& Boynton, R. M. (1978). Chromatic border perception: The role of red- and green-sensitive cones. Vision Research, 18, 683-697.
VALBERG, A., TANSLEY, B. W. (1977). Tritanopic purity-difference function to describe the properties of minimal distinct borders. Journal of the Optical Society of America, 67, 1330-1336.

WAGNER, W., \& BoYNTON, R. M. (1972). Comparison of four methods of heterochromatic photometry. Journal of the Optical Society of America, 62, 1508-1515.

WARD, F., B BoYNTON, R. M. (1974). Scaling of large chromatic differences. Vision Research, 14, 943-949.

Wittebrood, J. E. M., Wansink, M. G., d DE Weert, C. M. M. (1981). A versatile color stimulus generator. Perception, 10, 63-69. Wyszeck1, G., \& STILES, W. S. (1983). Color science. New York: Wiley.

\section{NOTE}

1. The discrepancy between the MDB and flicker criterion was unexpected, since Kaiser (1971) and Wagner and Boynton (1972) stated that both methods for luminance calibration are very similar. In a control experiment, with the same 4 subjects using the right eye only and 5 additional subjects, we found that a flicker photometric match with white of $10 \mathrm{~cd} / \mathrm{m}^{2}$ yields an average (9 subjects) blue and yellow luminance level of 10.5 and $10.0 \mathrm{~cd} / \mathrm{m}^{2}$, respectively. The MDB match of blue with yellow, with the yellow luminance fixed at $10 \mathrm{~cd} / \mathrm{m}^{2}$, yields an average ( 9 subjects) luminance of $13 \mathrm{~cd} / \mathrm{m}^{2}$ for blue. Thus we find that significantly more blue is needed in a MDB match than in the flicker match. In a control experiment, we found that the discrepancy disappears when artificial pupils and achromatizing lenses are used.

(Manuscript received October 26, 1987; revision accepted for publication May 25, 1988.) 